\title{
Nanoparticle Assemblies as Memristors
}

Tae Hee Kim, ${ }^{1 *}$ Eun Young Jang, ${ }^{1}$ Nyun Jong Lee, ${ }^{1}$ Deung Jang Choi, ${ }^{1}$ Kyung-Jin Lee, ${ }^{2}$ Jung-tak Jang, ${ }^{3}$ Jin-sil Choi, ${ }^{3}$ Seung Ho Moon, ${ }^{3}$ Jinwoo Cheon ${ }^{3} *$

\author{
${ }^{1}$ Department of Physics, Ewha Womans University, Seoul 120-750, Korea \\ ${ }^{2}$ Department of Materials Science and Engineering, Korea University, Seoul 136-713, Korea \\ ${ }^{3}$ Department of Chemistry, Yonsei University, Seoul 120-749, Korea
}

*correspondence authors: taehee@ewha.ac.kr; jcheon@yonsei.ac.kr 


\section{TEM images of size and composition controlled metal ferrite}

\section{$\left(\mathrm{MFe}_{2} \mathrm{O}_{4}, \mathrm{M}=\mathrm{Mn}, \mathrm{Fe}, \mathrm{Co}, \mathrm{Ni}\right)$ nanoparticles}

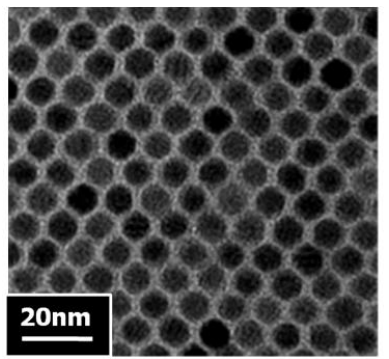

$7 \mathrm{~nm}$

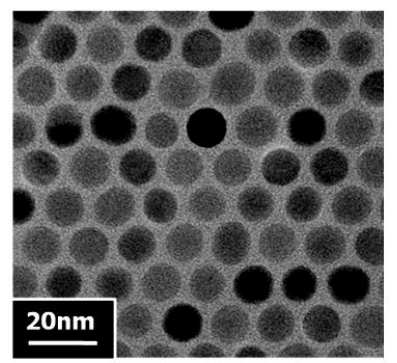

$9 \mathrm{~nm}$

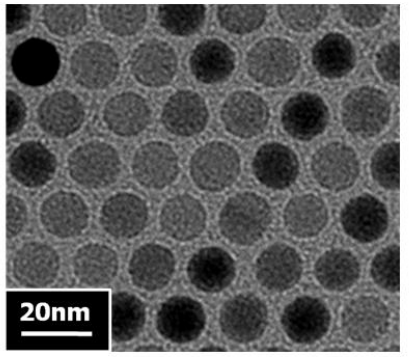

$12 \mathrm{~nm}$

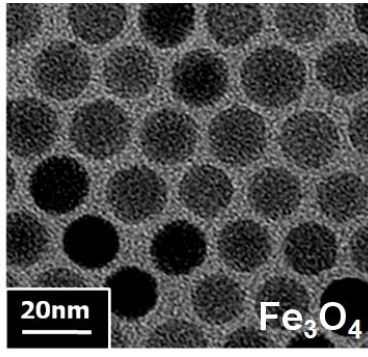

$15 \mathrm{~nm}$

Figure S1. TEM images of $\mathrm{Fe}_{3} \mathrm{O}_{4}$ nanoparticles with diameters of $7 \mathrm{~nm}, 9 \mathrm{~nm}, 12 \mathrm{~nm}$, and 15 $\mathrm{nm}$ with narrow distribution $(\sigma \approx 5 \%)$.

(a)

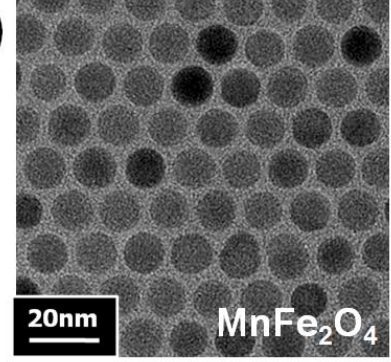

$12 \mathrm{~nm}$ (b)

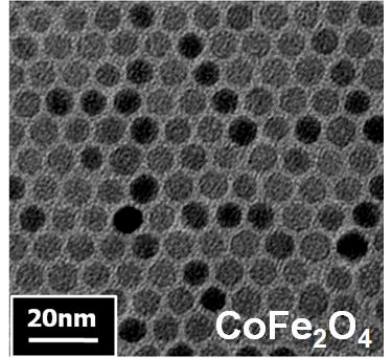

$7 n m$ (c)

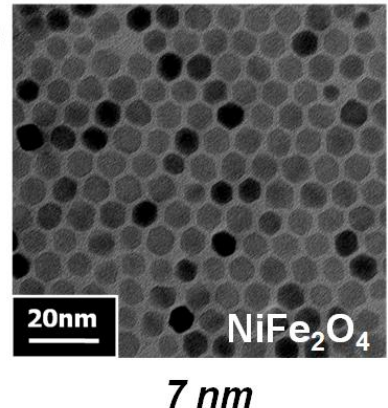

Figure S2. TEM images of (a) $12 \mathrm{~nm} \mathrm{MnFe}_{2} \mathrm{O}_{4}$, (b) $7 \mathrm{~nm} \mathrm{CoFe} \mathrm{O}_{4}$ and (c) $7 \mathrm{~nm} \mathrm{NiFe} \mathrm{O}_{4}$ nanoparticles with narrow distribution $(\sigma \approx 5 \%)$. 
Reversible switching behaviors of other spinel structured nanoparticles

(a)

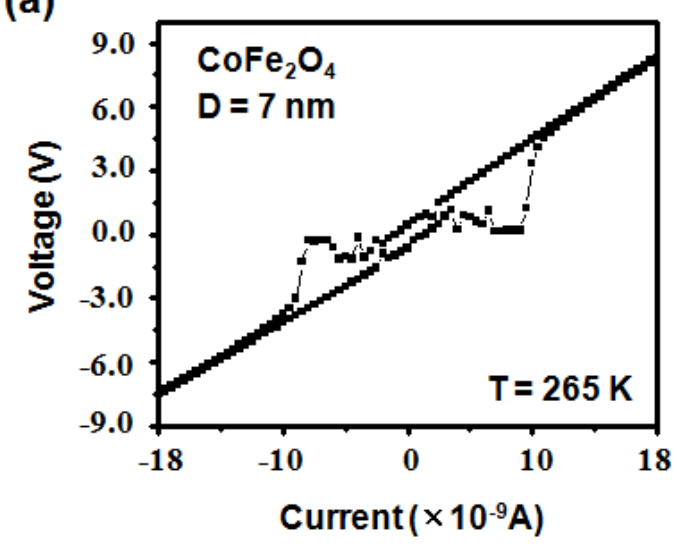

(b)

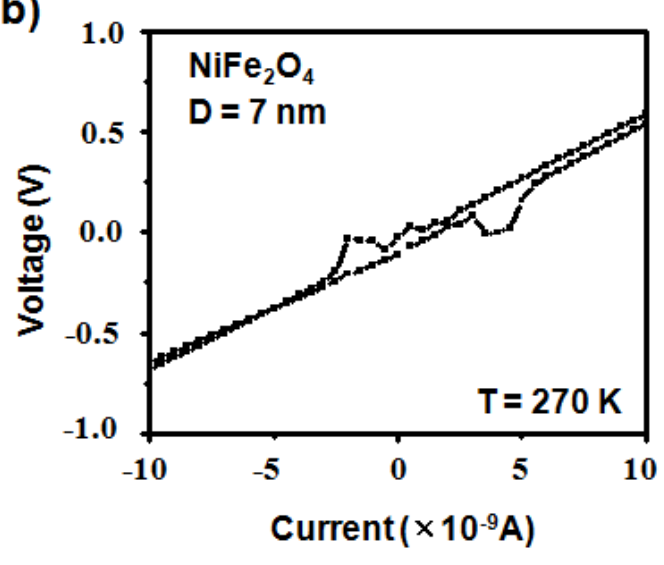

Figure S3. V-I characteristics of (a) $\mathrm{CoFe}_{2} \mathrm{O}_{4}$ and (b) $\mathrm{NiFe}_{2} \mathrm{O}_{4}$ nanoparticle assemblies, respectively. $7 \mathrm{~nm}$ sized $\mathrm{CoFe}_{2} \mathrm{O}_{4}$ and $\mathrm{NiFe}_{2} \mathrm{O}_{4}$ nanoparticle assemblies show switching behaviors at $265 \mathrm{~K}$ and $270 \mathrm{~K}$, respectively. 


\section{TEM images of a pellet $\left(\mathrm{Fe}_{3} \mathrm{O}_{4}\right.$ nanoparticle assemblies)}

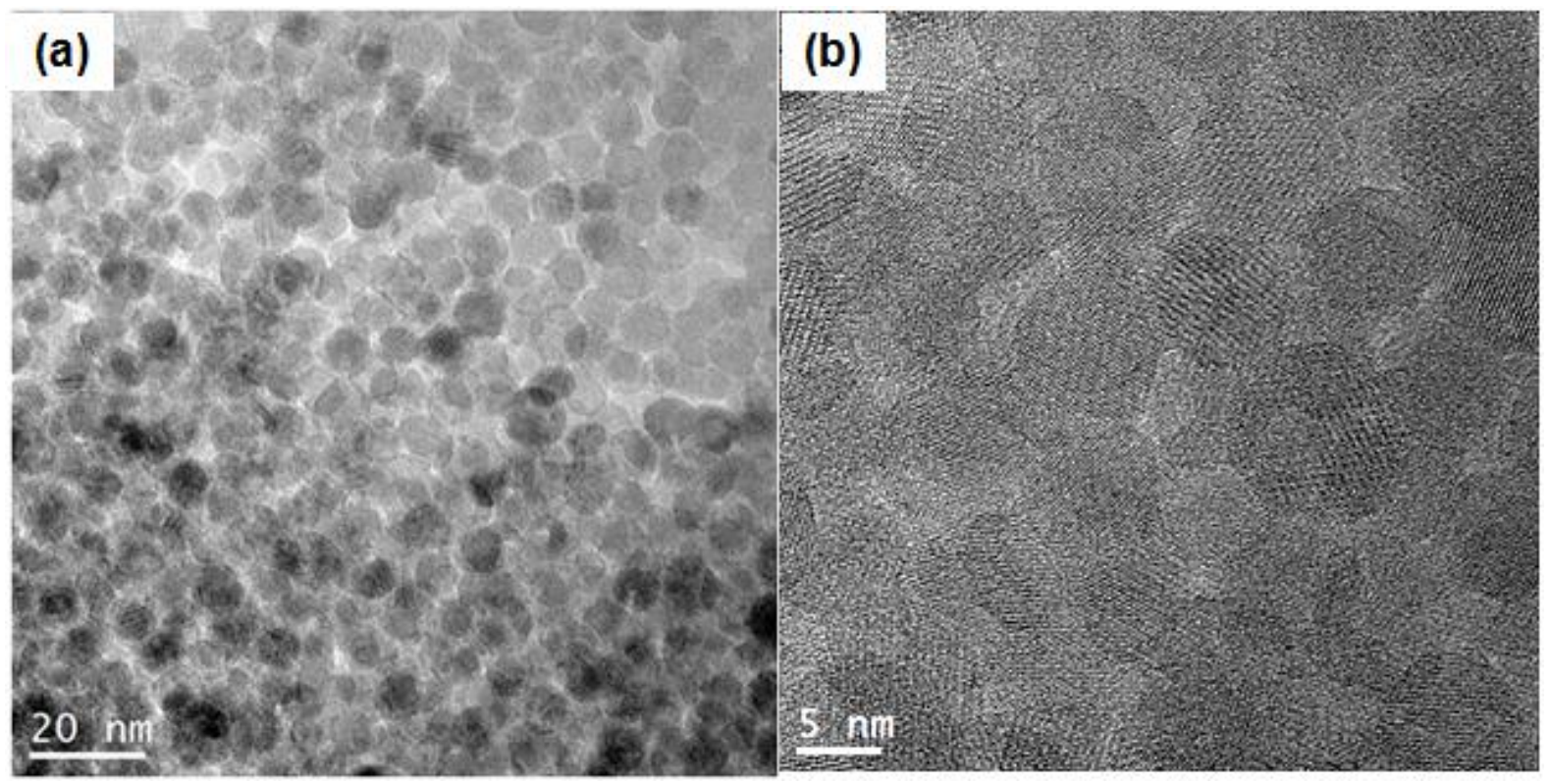

Figure S4. (a, b) TEM images of a pellet of $7 \mathrm{~nm}$ sized $\mathrm{Fe}_{3} \mathrm{O}_{4}$ nanoparticle assemblies at low and high magnification, respectively. 